\title{
Clinical Profiles of Children (0 - 18 Years Old and 364 days) with Coronavirus Disease 2019 (COVID 19) at St. Luke's Medical Center Quezon City
}

\section{Arvee Riza R Aquilizan*, Elynn L Go and Imelda Luna}

Institute of Pediatrics and Child Health, St. Luke's Medical Center, Quezon City,

Philippines

*Corresponding Author: Arvee Riza R Aquilizan, Institute of Pediatrics and Child Health, St. Luke's Medical Center, Quezon City, Philippines.

DOI: 10.31080/ASPE.2021.04.0455
Received: July 27, 2021

Published: September 24, 2021

(C) All rights are reserved by Arvee Riza $\mathbf{R}$

Aquilizan., et al.

\section{Abstract}

Background: Coronavirus Disease 2019 (COVID-19) has become a worldwide dilemma still with increasing number of pediatric cases reported. Thus, it is important to know the clinical profile of these pediatric patients to further understand its course and outcome of management.

Objective: To describe the clinical profiles of confirmed COVID-19 pediatric patients at St. Luke's Medical Center Quezon City from March to September 2020.

Design: This research is a descriptive cross-sectional study.

Subject: This study included all pediatric patients (0 - 18 years and 364 days old) confirmed COVID- 19 at St. Luke's Medical Center Quezon City.

Methodology: A review of the electronic and written medical records was conducted.

Data analysis: Data will be presented as descriptive statistics using frequencies, proportions, means and standard deviation.

Results: A total of 90 pediatric patients were included. Majority of the children belonged to the 11 - 15 years age group and $76 \%$ were seen at the emergency department. Forty-six (51\%) of them were female and forty-four (48.9\%) were male. Most of the patients (59 patients, 68.6\%) had known exposure to confirmed family members. Sixty-two of the subjects (68.9\%) had mild signs and symptoms while twenty-five (27.8\%) were asymptomatic. The most common symptoms seen in our subjects were fever (40\%), and respiratory symptoms like cough (30\%) and rhinorrhea (22.2\%). Other notable symptoms were anosmia (8.9\%), and dysgeusia (6.7\%). Most of the children had normal chest x-ray findings (60.7\%). In the laboratory findings of these patients, it was noted that majority had neutrophilia (74.2\%). Almost all of the patients were discharged (98.9\%) and was given supportive management.

Conclusion: Children with COVID-19 presented with milder clinical course and rarely we encounter critical cases compared to infected adults. Early detection is the key to prevent further spread of this disease but a lot of our patients were asymptomatic hence diagnosis is a challenge for pediatricians. Proper quarantine precautions must be emphasized to primary caregivers of these patients. Keywords: COVID-19; Pediatric; Children; Clinical Profiles; Epidemiology

\section{Introduction}

Significance of the project

Coronavirus disease 2019 (COVID-19) caused by severe acute respiratory syndrome coronavirus 2 (SARS- COV-2), a novel corona virus, was declared a pandemic last March 11, 2020 by the World Health Organization (WHO) as it had spread around the world. Initially, it was thought that it may only affect adults but the number of pediatric patients being affected continue to increase with varying 
manifestations not seen in adults. Data regarding the clinical characteristics of these pediatric patients are lacking in the Philippines.

Rationale for doing the study

COVID-19 is a relatively new disease with increasing number of pediatric cases being reported. Early detection is the key to prevent further increase and spread of the cases. It is quite important to know the clinical profile and demographics of pediatric patients infected with this virus to further understand its clinical course and outcome which will help us prevent and manage this disease.

Background information and brief literature review

SARS- CoV-2, the etiology agent of coronavirus disease has spread worldwide, resulting to a global public health threat. This virus was unknown before the outbreak began in Wuhan, China, in December 2019.

Globally, as of January 2021, a total of 100 million cases of COVID- 19 with a million of deaths were listed by the WHO [21]. The Philippines already has estimated 518,407 cases with 10,481 deaths recorded as of January 2021 also and the number is still continuously rising daily [8].

The first case reported was a 38-year-old female Chinese national. Local transmission followed suit on March 7, 2020. The WHO then started working closely with the Philippine Department of Health in addressing this COVID-19 outbreak [23]. Approximately 46 thousand Filipino children ( $9 \%$ of the total count) have been diagnosed with this disease as of January 2021 with around 1\% fatality rate based on the Philippine DOH Tracker of the cases [8].

\section{Pediatric COVID-19 cases}

Worldwide, there were reported pediatric cases in various parts of the world. In the United States, $2 \%$ of confirmed cases of COVID-19 were among persons aged $<18$ years [3]. While in China, $2.2 \%$ of confirmed cases of COVID-19 were among persons aged $<19$ years old [24]. In Italy, 1.2\% of COVID-19 cases were among children aged < 18 years [19]. In Spain, $0.8 \%$ of confirmed cases of COVID-19 were among persons aged $<18$ years [3,19,22,24].

The first pediatric patient infected with COVID-19 in the Philippines was reported last March 16, 2020 at the Quirino Memorial Medical Center. This was a 13-year-old female who presented with cough and colds admitted for a few days eventually improved and was discharged.
As with the adults, the incubation period for the human infection in children ranges from 2 - 14 days according to the Center for Disease Control and Prevention (CDC).

Studies conducted in China, Korea and the US found out that majority of the children with confirmed COVID-19 had a household contact to a suspected or confirmed COVID- 19 patient [11-13,25]. Han., et al. in 2020 conducted a study on the clinical characteristics of COVID-19 in Korea and they found out that the most common source of infection was household contact (63\%), followed by importation (17\%), cluster-associated transmission (12\%), other contacts (4\%), and unknown sources (4\%) [12].

\section{Clinical presentation}

The CDC included fever, cough, nasal congestion or rhinorrhea, sore throat, shortness of breath, diarrhea, nausea or vomiting, fatigue, headache, myalgia and poor feeding or poor appetite as the signs and symptoms over the course of the disease for pediatric patients with COVID-19 [7]. Furthermore, the clinical presentations seen in children with this disease were similar to other viral respiratory infections, including fever, cough, and shortness of breath although these signs and symptoms may occur at any time during the overall disease course [7]. Children with COVID-19 may not initially present with fever and cough as often as adult patients in fact, in a study done by Ji., et al. diarrhea was the only symptom reported in one of the pediatric case of COVID-19 [17].

On a systematic review done by Hoang., et al. on pediatric COVID 19 patients, they summarized the clinical symptom findings in COVID-19 confirmed pediatric patients. No symptoms were described in 456 of 2367 patients (19.3\%), while the two most common symptoms were fever (59.1\%), and cough (55.9\%). Upper respiratory symptoms were the characteristic of presentation of COVID-19 in children, some patients also presented with mild or often overlooked symptoms such as fatigue, abdominal pain, or decreased appetite [13].

Chang., et al. also conducted a systematic review and meta-analysis on COVID 19 children and similarly majority of children (98\%) were categorized as having mild to moderate disease severity. 59\% of the patients presented with fever while $46 \%$ had a cough [4].

\section{Clinical course in children}

The largest study of pediatric patients $(>2,000)$ with COVID-19 from China reported that illness severity ranged from asymptom- 
atic to critical $[7,10]$. The following are the definitions of the various categories of the clinical course: a. Asymptomatic (4\%) where there are no clinical signs or symptoms with normal chest imaging. b. Mild (51\%) where symptoms include fever, fatigue, myalgia, cough; c. Moderate (39\%) where there is pneumonia with symptoms or subclinical disease with abnormal chest imaging; d. Severe (5\%) where symptoms include dyspnea, central cyanosis, hypoxia; e. Critical $(0.6 \%)$ where it includes acute respiratory distress syndrome [ARDS], respiratory failure, shock, or multi-organ dysfunction $[7,10]$.

Results from a study done, "Epidemiology of COVID-19 among Children in China," by Dong Y., et al. concluded that pediatric patients in at all ages were susceptible to COVID-19, and that there was no significant difference between genders. Less severe clinical manifestations were also seen in children than those of adults' patients. However, young children, particularly infants, were vulnerable to COVID-19 infection [10].

\section{Radiographic and laboratory tests}

Common radiographic findings seen in children with COVID-19 were patchy consolidation (31\%) and ground glass opacities (48\%) as noted on the study by Chang., et al. in Taiwan [13]. Typical laboratory findings being seen according to CDC were mild changes in white blood cell count (either increased or decreased lymphocyte counts), mildly elevated inflammatory markers (including procalcitonin, c-reactive protein), and mildly elevated liver enzymes [7].

Reverse transcriptase polymerase chain reaction as diagnostic test for covid-19

The IDSA (Infectious Disease Society of America) and CDC described SARS-CoV-2 reverse transcriptase-polymerase chain reaction (RT-PCR) as a test that detects viral RNA, and a positive result is highly specific for the presence of the virus. There are a lot of specimen that can be used for RT- PCR. It could be via oronasal swab, nasopharyngeal swab, laryngeotracheobronchial aspirates, sputum, and saliva. Most commonly, we used the combination of oropharyngeal and nasopharyngeal swab in testing suspected cases of COVID- 19 as advised by the Department of Health which we similarly utilize at St. Luke's Medical Center [16].

Different studies conducted had shown that the RT-PCR has a sensitivity of $73-95 \%$ in the detecting the viral RNA for the di- agnosis of COVID-19 [6,14,21]. A study by Holborow., et al. on the clinical sensitivity of a single SARS-CoV-2 upper respiratory tract RT-PCR test for diagnosing COVID-19 found out that the sensitivity in symptomatic individuals at $86.2 \%$ while the clinical false negative rate of a single RT-PCR on an upper respiratory tract sample of $14 \%$ in symptomatic patient [14]. Comparing it to a study done by Corman., et al. with a sensitivity of 95\% [6].

According to the interim guidelines on the screening, assessment and clinical management of pediatric patients with suspected or confirmed COVID-19 by the Philippine Pediatric Society (PPS) and Pediatric Infectious Disease Society of the Philippines (PIDSP) last 2020, in order to confirm the diagnosis of this disease, detection of the causative agent SARS-CoV-2 is needed using nucleic acid testing such as RT-PCR or other PCR-based test. The preferred specimen for this test is nasopharyngeal swab but oropharyngeal swab may be added [16]. They further included in the guideline that once a suspect COVID-19 case is identified, appropriate respiratory specimens should be collected as soon as possible regardless of the time of symptom onset as what was advised by WHO [16].

In the study done by Han., et al. in Korea, their cases of COVID-19 were similarly diagnosed by detecting SARS-CoV-2 RNA in a combined nasopharyngeal and oropharyngeal swab or sputum by RT-PCR [12].

\section{Treatment outcomes}

In the management of children infected with COVID- 19, we need to consider the clinical presentation, underlying comorbidities, the severity of the disease, and the ability and capacity of the primary caregiver to provide home management. According to CDC, PPS and PIDSP, treatment for pediatric COVID-19 is largely supportive [7]. The CDC also mentioned that there are no drugs specifically approved by the U.S. Food and Drug Administration (FDA) for treatment of COVID-19 in children [7].

PPS and PIDSP stated that antiviral medications are not yet proven to be effective in treating this disease and currently, they only recommend use of medications for severe suspected, probable, or confirmed COVID-19 cases. Remedesivir is an experimental drug for compassionate use that may be given for severe and critical COVID. They also included Dexamethasone, Tocilizumab, and intravenous immunoglobulin as adjunctive therapies. Other medi- 
cations mentioned were Zinc sulfate and Vitamin D3 [15]. These therapeutic interventions are still evolving as more data from other researches are coming in.

In the study done by Han., et al. in Korea, $85 \%$ of the children with COVID-19 did not receive any management. Regardless of what was mentioned in the guidelines, this study noted $13 \%$ of the children were treated with lopinavir-ritonavir, and $2 \%$ received hydroxychloroquine. One patient (1\%) received both lopinavir-ritonavir and hydroxychloroquine. Among these patients who received treatment, there was no difference in the mean duration of virus RNA detection between patients treated with lopinavir-ritonavir vs without lopinavir-ritonavir (17.9 [4.5] days vs 17.3 [8.3] days; $\mathrm{P}=$ .79). All had good prognosis and no fatality case was noted [12].

\section{General objective}

To describe the clinical profiles of confirmed COVID-19 pediatric patients diagnosed at St Luke's Medical Center from March to September 2020.

\section{Specific objectives}

- To report the following demographic factors: (a) age, (c) sex.

- To determine these clinical characteristics: (a) initial Diagnosis, (b) final Diagnosis, (c) exposure or contact information, (d) comorbidities, (e) signs and symptoms, (f) illness severity.

- To describe the following laboratory and diagnostic tests: (a) chest x-ray, (b) CBC, (c) ESR, (d) CRP, (e)Procalcitonin, (f) Blood Culture, (g) ABG/VBG, (h) LDH, (i)Ferritin, (j) Others.

- To determine the treatment modalities, and outcome of these children with COVID 19.

\section{Methods}

Type of study, time period and target population

This research was a descriptive, cross sectional study. A review of the electronic and written medical records was conducted from March to September 2020. This study includes all pediatric patients (0 - 18 years and 364 days old) confirmed to have COVID-19 at St. Luke's Medical Center Quezon City. Data were extracted from patients' medical records. Data collection was conducted through chart review of medical charts.

\section{Criteria for subject selection}

The inclusion criteria were as follows:

1. Ages 0 - 18 years and 364 days old.

2. Seen at the ER (discharged), admitted from the ER, newborns born at SLMC, inpatients diagnosed while hospitalized.

3. Confirmed COVID-19 positive by COVID Test RT-PCR at St. Luke's Medical Center Quezon City.

The exclusion criteria were as follows:

1. Patients with incomplete data.

Method of subject selection

Medical records of pediatric patients from March to September 30, 2020 were included in the review. Patients who consulted at the E.R., discharged, or admitted, newborns, in-patients diagnosed at the ward while hospitalized.

Data to be gathered

The following data were included in the study:

1. Age.

2. Sex.

3. Initial diagnosis.

4. Final diagnosis.

5. Exposure or contact information:

a. Family cluster:

i. Confirmed family members.

ii. Suspected family members.

b. Unidentified source of infection.

c. Contact with other suspected case.

6. Comorbidities:
a. Cancer.
b. Hypertension.
c. Others.

7. Signs and symptoms
a. Cough.
b. Pharyngeal erythema.
c. Fever. 
d. Duration of fever.

e. Highest temperature during hospitalization $\left({ }^{\circ} \mathrm{C}\right)$ :

$$
\text { i. } \quad<37.5 \text {. }
$$

ii. $\quad 37.5$ - 38 .

iii. $\quad 38.1$ - 39 .

iv. $>39$.

f. Diarrhea.

g. Nasal congestion.

h. Tachypnea on admission.

i. Tachycardia on admission.

j. Oxygen saturation $<92 \%$ during period of hospitalization.

k. Shortness of breath or difficulty of breathing.

l. Rashes.

m. Anosmia.

n. Dysgeusia.

o. Others.

8. Illness severity [11]:

a. Asymptomatic defined as no clinical signs or symptoms with normal chest imaging [11].

b. Mild defined as mild symptoms, including fever, fatigue, myalgia, cough [11].

c. Moderate defined as pneumonia with symptoms or subclinical disease with abnormal chest imaging [11].

d. Severe defined as dyspnea, central cyanosis, hypoxia [11].

e. Critical defined as acute respiratory distress syndrome [ARDS], respiratory failure, shock, or multi-organ dysfunction [11].

9. Radiographic findings:
a. Ground glass opacity.
b. Local patchy shadowing.
c. Bilateral patchy shadowing.
d. Interstitial abnormalities.
e. Others.

10. Laboratory tests:
a. $\mathrm{CBC}$.
b. ESR.

c. CRP.

d. Procalcitonin.

e. Blood culture.

f. $A B G / V B G$.

g. LDH.

h. Ferritin.

i. Others.

11. Treatment

a. Antibiotics.

b. Antiviral.

c. Oxygen support.

d. Supportive management.

e. Observation.

f. Others.

12. ICU admission

13. Outcome
a. Discharged.
b. Expired.

Sample size estimation

All pediatric patients ( 0 - 18 years and 364 days old) confirmed COVID-19 at St. Luke's Medical Center Quezon City from March to September 2020 were included.

\section{Study procedures}

All confirmed COVID-19 pediatric patients who tested positive using RT PCR were recruited and included in the study. Review of charts of these patients was done by using the database of the hospital. Demographic profile, clinical characteristics, laboratory and diagnostic abnormalities, and mortality or morbidity of the patients were also reviewed. All data were recorded.

\section{Outcomes}

Our outcome of interest included demographic profile, clinical characteristics, laboratory and diagnostic tests abnormality, and morbidities and mortality of the confirmed COVID-19 pediatric patients.

Data analysis

Descriptive statistics were used to present the data. Mean and 
standard deviation were used for the quantitative variables while frequencies and proportion on the categorical variables. Categorical variables were further expressed as counts and percentage.

Operational definitions

- Coronavirus disease 2019 (COVID-19): A viral infectious disease caused by a novel strain of coronavirus, SARS COV-2.

- Coronavirus disease 2019 (COVID-19) positive or confirmed: Patients with positive or confirmed COVID-19 with nasopharyngeal and oropharyngeal COVID RT-PCR.

- $\quad$ Real time RT-PCR: The diagnostic test of choice for the confirmation of SARS-COV-2 infection.

- Asymptomatic infection: No clinical signs and symptoms; \pm chest imaging is normal, real time RT-PCR for SARS-COV-2 is positive.

- Leukopenia: Low white cell count based on normal values per age [14].

- Leukocytosis: High white cell count based on normal values per age [14].

- Lymphocytic: Predominance of lymphocytes in the blood based on normal values per age [14].

- Neutrophilic/Neutrophilia: Predominance of neutrophils in the blood based on normal values per age [14].

- Lymphopenia: Low lymphocyte count in the blood based on normal values per age [14].

- Thrombocytopenia: Low platelet count in the blood based on normal values per age [14].

- Thrombocytosis: High platelet count in the blood based on normal values per age [14].

\section{Ethical considerations}

The research protocol and any relevant documents was submitted to the St. Luke's Medical Center Quezon City Ethics Review Board/Committee and have followed the guidelines set by the Philippine Health Research Ethics Board (PHREB), whereby, this research paper was ensured with the following.

First, the study abided the Principles of the Declaration of Hel- sinki (2013) and was conducted along the Guidelines of the International Conference on Harmonization-Good Clinical Practice (ICH-GCP). Second, the Clinical Protocol and all relevant documents was reviewed and approved by the SLMC Institutional Ethics Review Committee. Third, patient confidentiality was respected by ensuring anonymity of patient records. Fourth, each patient document was CODED and did not contain any identifying information to ensure confidentiality. Fifth, all study data was recorded, and investigators were responsible for the integrity of the data i.e. accuracy, completeness, legibility, originality, timeliness and consistency. Sixth, the manner of disseminating and communicating the study results guaranteed the protection of the confidentiality of patient's data. Lastly, all study-related documents such as all versions of the protocol, ethical clearance, data collection forms, hard copies of source documents were kept and stored by the Principal Investigator in strict confidentiality for at least 5 years; after which they will be shredded.

\section{Results}

Demographic characteristics of pediatric COVID- 19

A total of 90 subjects were included in the study. Table 1 shows the demographic characteristics of pediatric patients with COVID 19. The mean age was 5.78 (6.2) and ranges from 0 to 18 years. Majority of the children belonged to the 11 - 15 years age group. There were more female children infected with COVID-19 than males.

\begin{tabular}{|l|c|}
\hline Age group & MeanSD \\
\hline & 5.786 .2 \\
\hline Age group n = 90 & $\mathbf{n}(\%)$ \\
\hline$<1$ year & $13(14.4)$ \\
\hline 1 - 5 yrs & $20(22.2)$ \\
\hline 6 -10 yrs & $18(20.0)$ \\
\hline $11-15$ yrs & $22(24.4)$ \\
\hline $16-18$ yrs & $17(18.9)$ \\
\hline Sex $\mathbf{n}=\mathbf{9 0}$ & $\mathbf{n}(\%)$ \\
\hline Male & $44(48.9)$ \\
\hline Female & $46(51.1)$ \\
\hline
\end{tabular}

Table 1: Demographic characteristics of pediatric COVID-19.

\section{Clinical characteristics of pediatric COVID-19}

Majority (76.7\%) were seen at the emergency department. We had $12.2 \%$ newborns with COVID 19. Most of the patients (68.6\%) 
had known exposure to confirmed family members. Among the children infected with SARS COV- 2, 70.4\% had no comorbidities.

\begin{tabular}{|l|c|}
\hline Clinical Characteristics $\mathbf{n}=\mathbf{9 0}$ & $\mathbf{n}(\mathbf{\%})$ \\
\hline Seen at ER and discharged & $69(76.7)$ \\
\hline Admitted & $21(23.3)$ \\
\hline Admitted from the ER & $8(8.9)$ \\
\hline Newborns & $11(12.2)$ \\
\hline Inpatient diagnose & $2(2.2)$ \\
\hline Exposure $\mathbf{n}=\mathbf{8 6}$ & \\
\hline Confirmed family members & $59(68.6)$ \\
\hline Unidentified source of infection & $18(20.9)$ \\
\hline Suspected family members & $9(10.5)$ \\
\hline Contact with other suspected case & 0 \\
\hline Comorbidities n = 52 & \\
\hline None & $38(70.4)$ \\
\hline Bronchial Asthma & $6(11.1)$ \\
\hline Cancer & $2(3.7)$ \\
\hline Allergic rhinitis & $2(3.7)$ \\
\hline Prematurity & $1(1.8)$ \\
\hline Hypertension & 0 \\
\hline Others & $5(9.3)$ \\
\hline Neurogenic bladder (1) & \\
\hline Arthrogryposis multiplex congenital (1) & \\
\hline Urinary Tract Infection (1) & \\
\hline Autism Spectrum Disorder (1) & \\
\hline Complex Febrile Seizure (1) & \\
\hline Gastroesophageal reflux disease (1) & \\
\hline
\end{tabular}

Table 2a: Clinical characteristics of pediatric patients with COVID 19.

\section{Illness severity}

On table $2 \mathrm{~b}$, among the 90 pediatric patients with COVID-19, majority (68.9\%) had mild signs and symptoms while $27.8 \%$ were asymptomatic.

\begin{tabular}{|l|c|}
\hline Severity N = 90 & n (\%) \\
\hline Asymptomatic & $25(27.8)$ \\
\hline Mild & $62(68.9)$ \\
\hline Moderate & 0 \\
\hline Severe & $2(2.2)$ \\
\hline Critical & $1(1.1)$ \\
\hline
\end{tabular}

Table 2b: Illness severity of the children with COVID-19.

\section{Clinical signs and symptoms}

Table $2 c$ shows that $40 \%$ of the subjects developed fever among them, majority (76\%) lasted for 1-2 days only. Highest fever recorded ranges from $38.1-39^{\circ} \mathrm{C}$. Other common symptom noted were respiratory symptoms. $30 \%$ of children had cough and $22.2 \%$ had rhinorrhea.

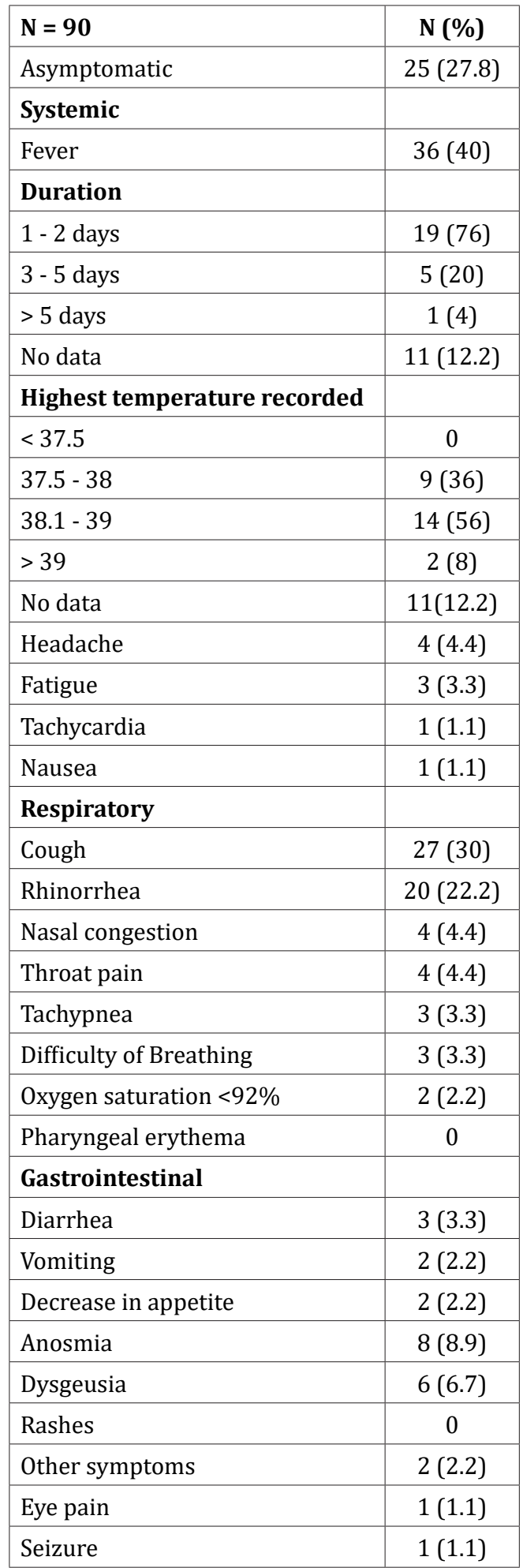

Table 2c: Clinical manifestations of children with COVID-19. 


\section{Radiologic findings}

Among the 28 patients with COVID-19 who had their chest $\mathrm{x}$ rays, majority $(60.7 \%)$ had normal findings. Twenty five percent $(25 \%)$ had bilateral patchy shadowing and the rest had local patchy shadowing (10.7\%).

\begin{tabular}{|l|c|}
\hline Chest X-ray Findings $\mathbf{n}=\mathbf{2 8}$ & $\mathbf{n}(\mathbf{\%})$ \\
\hline Few tiny nodular densities & $1(3.6)$ \\
\hline Local patchy shadowing & $3(10.7)$ \\
\hline Bilateral patchy shadowing & $7(25)$ \\
\hline Normal & $17(60.7)$ \\
\hline
\end{tabular}

Table 3a: Chest X-ray findings of pediatric patients with COVID-19.

\section{Laboratory findings}

As shown in table 3b, 31 children with COVID 19 had their complete blood counts. White blood cell count was normal in most of the pediatric patients $(67.7 \%)$. In terms of leukocyte predominance, majority $(74.2 \%, n=23)$ had neutrophilia. Some of the patients presented with more than $1 \mathrm{WBC}$ type. Platelet counts were normal on $83.9 \%(n=26)$ of the patients. Other laboratory tests done in table $3 \mathrm{c}$ showed that the inflammatory markers were not elevated. Only $2 \%(n=2)$ of those tested with C-reactive protein had abnormal findings while children who had their procalcitonin and blood cultures were all normal. One patient in particular had abnormal ferritin. Ferritin was requested to rule out other causes of neuroleptic malignant syndrome comorbidity of the patient.

\begin{tabular}{|l|c|}
\hline White blood count $\mathbf{n}=\mathbf{3 1}$ & $\mathbf{n}(\%)$ \\
\hline Normal & $21(67.7)$ \\
\hline Leukopenia & $5(16.1)$ \\
\hline Leukocytosis & $5(16.1)$ \\
\hline WBC Type & $\mathrm{n}(\%)$ \\
\hline Neutrophilic & $23(74.2)$ \\
\hline Lymphocytic & $7(22.6)$ \\
\hline Immature cells & $4(12.9)$ \\
\hline Lymphopenia & $4(12.9)$ \\
\hline Platelet n = 31 & $\mathbf{n}(\%)$ \\
\hline Normal & $26(83.9)$ \\
\hline Thrombocytopenia & $3(9.7)$ \\
\hline Thrombocytosis & $2(6.5)$ \\
\hline
\end{tabular}

Table 3b: Complete blood count findings of patients with COVID-19.

\begin{tabular}{|l|c|c|}
\hline & $\begin{array}{c}\text { Abnormal } \\
\text { n (\%) }\end{array}$ & $\begin{array}{c}\text { Normal } \\
\text { n (\%) }\end{array}$ \\
\hline C-reactive protein $(\mathrm{n}=8)$ & $2(25)$ & $6(75)$ \\
\hline ESR (n=0) & 0 & 0 \\
\hline Procalcitonin (n=3) & 0 & $3(100)$ \\
\hline Blood CS (n=9) & 0 & $9(100)$ \\
\hline Venous blood gas $(\mathrm{n}=3)$ & $2(66.7)$ & $1(33.3)$ \\
\hline Lactate dehydrogenase $(\mathrm{n}=1)$ & 0 & $1(100)$ \\
\hline Ferritin $(\mathrm{n}=1)$ & $1(100)$ & 0 \\
\hline
\end{tabular}

Table 3c: Other Laboratory tests findings of pediatric children with COVID-19.

Treatment and outcome

On table 4, majority (85.6\%) of the children with COVID-19 were given supportive management, and about $14.4 \% \quad(n=13)$ received antibiotics. Different antibiotics were given to these patients. There were 5 neonates with the consideration of early/late onset sepsis, hence they were given Ampicillin and Gentamicin/Cefotaxime. While on the remaining patients with antibiotics, 4 were given Azithromycin after noting neutrophilia hence bacterial coinfection was contemplated on these patients. The other remaining patients were given antibiotics (Cefuroxime, Ticarcillin Clavulanic Acid, Cefalexin) had comorbidities of other bacterial infections like urinary tract infection, wound infection and another one also had immunodeficiency secondary to leukemia. For the patients who received respiratory support, 3.3\% were given oxygen support, and $2.2 \%$ were hooked to a mechanical ventilator. A total of $4(4.4 \%)$ of children with COVID-19 were transferred to intensive care unit. The indications for the transfer to the ICU was due to their comorbidities. Two of the critical patients were newborns presented with respiratory distress and were eventually intubated, another one had a comorbidity of acute lymphocytic leukemia relapse with complications of pneumonia while the last one was an adolescent who needs closer monitoring because of neuroleptic malignant syndrome secondary to intentional substance ingestion (Fluoxetine, Clozapine), intentional. We had 1 mortality case who had a co morbidity of malignancy (Acute lymphocytic leukemia in relapse).

\section{Discussion}

According to the WHO as of December 27, 2020, over 78 million worldwide cases of COVID-19 infection have been noted and our knowledge of the disease and its epidemiologic and clinical char- 
acteristics continue to evolve. Locally, continuous studies and data checking have been done by DOH to monitor the increasing cases still for this disease. In this study, we focused with the demographic features of children infected with SARS COV 2.

\begin{tabular}{|l|c|}
\hline Treatments $\mathbf{n}=\mathbf{9 0}$ & $\mathbf{n}(\%)$ \\
\hline Antibiotic & $13(14.4)$ \\
\hline Supportive management & $77(85.6)$ \\
\hline Anti-viral & 0 \\
\hline Respiratory Support $\mathbf{n}=\mathbf{9 0}$ & $\mathbf{n}(\%)$ \\
\hline None & $85(94.4)$ \\
\hline Oxygen Support & $3(3.3)$ \\
\hline Mechanical ventilator & $2(2.2)$ \\
\hline Intensive Care Management $\mathbf{n}=\mathbf{9 0}$ & $\mathbf{n}(\%)$ \\
\hline Yes & $4(4.4)$ \\
\hline No & $86(95.56)$ \\
\hline Outcome $\mathbf{n}=\mathbf{9 0}$ & $\mathbf{n}(\%)$ \\
\hline Discharged & $89(98.9)$ \\
\hline Expired & $1(1.1)$ \\
\hline
\end{tabular}

Table 4: Treatment and outcome of pediatric patients with COVID-19.

This study's findings may have several implications to our clinical practice. We found out that in this study that predominance is slightly more common in girls compared to boys (51.1\% vs $4.9 \%$ ) which is in contrast to a study done in China by Dong., et al. where $56.6 \%$ of their subjects were boys and $43.4 \%$ were girls [10]. This is also in contrast with the study done in Korea where $58 \%$ of their subjects were male while female had a prevalence of $42 \%$ [12]. The median age for these children infected with SARS COV- 2 was 8.5 years but ranges from newborns to 18 years of age. Most of the children infected with SARS-COV-2 have known household contact with a confirmed (68.6\%)/suspected (10.5\%) family member same as the recent studies done in Korea, China, and US [11-13,25]. In 2 systematic studies done in Houston, Texas and China, these studies both found that $75 \%$ of the pediatric patients with COVID19 had exposure to a family member with confirmed COVID-19 diagnosis [11,13].
In this study, most of the pediatric patients infected with SARS COV-2 presented with mild clinical manifestations (68.9\%) followed by the asymptomatic cases (27.8\%). This is comparable to the studies done in Korea, England, China, and US [10,11,13,20]. We may therefore conclude that these asymptomatic pediatric patients may have played a role in the transmission or spread of COVID-19 in the community. There were only a few $(4.4 \%)$ of the patients who were admitted at the intensive care unit and 1 mortality case in this study. These patients had co-morbidities like malignancy, and prematurity. One of our patients with acute myeloblastic leukemia recovered from this disease while the other one died. The latter was admitted in the hospital for 4 months already but was exposed to his father with confirmed infection. Progression to acute respiratory distress syndrome needing intensive care is a rarity in children with this disease in contrast to adults. Bronchial asthma (11.1\%) is the most common co morbidity seen among the subjects but was not contributory factor in the admission to the intensive care unit.

Children with COVID-19 present with variety of signs and symptoms which were nonspecific hence, not that helpful in the diagnosis of this disease and may be overlooked by pediatricians. In a systematic review and meta- analysis done involving 9 case series in Taiwan, approximately $59 \%$ of their subjects presented with fever and around 46\% had cough [11]. This is aligned to our study where the most common symptoms seen in our subjects were fever (40\%), and respiratory symptoms like cough (30\%) and rhinorrhea (22.2\%). Other notable symptoms were anosmia (8.9\%), and dysgeusia (6.7\%). Nevertheless, in adults we found a study in Germany by Lechien., et al. where they found out that olfactory $(85.6 \%)$ and gustatory dysfunctions (88.8\%) have a high prevalence as a clinical manifestation in COVID-19 which is in contrast with our study findings in children [18]. Since symptoms are nonspecific, confirming it through the use of RT PCR would be very helpful for infection control purposes and anticipation of possible complications.

Radiographic tests and laboratory tests like chest x-rays, complete blood count and inflammatory markers were done in a few of our subjects since in our country we always emphasize that we do diagnostics which were cost-effective, will play a role diagnosis and 
will be helpful in our management [16]. Most of our subjects had normal chest $\mathrm{x}$-rays $(60.7 \%)$ and only a few had opacities accounting to $35.7 \%$. This can be expected as majority did not present with any symptoms particularly respiratory in nature. In line with this is the systematic review on COVID-19 in 7780 pediatric patients done in Houston Texas, where they also found that majority of their subjects had unremarkable chest x-ray findings around 23\% [13]. Although in one of the studies done in the England about SARS COV-2 in children, they found out that asymptomatic children had radiographic features of pneumonia [20]. The above findings show that radiographic findings are not specific for COVID but would be helpful in classifying the severity of the disease. On the laboratory tests done to our patients, we found out that instead of lymphocyte predominance or lymphopenia in white cell counts as seen in the studies done in the US and China, in our study we particularly noted neutrophilia (74.2\%) among the patients with complete blood count tests $[11,13]$. In connection to this, a review done by Borges., et al. on COVID-19 and neutrophils where they discussed the relationship between hyperinflammation and neutrophil extracellular traps as seen in viral infections such as COVID 19 [26]. They also mentioned about different studies where several COVID 19 patients had increasing neutrophilic counts but were seen in severe cases of this disease [26]. Aside from this, the inflammatory markers like CRP and procalcitonin were not elevated in our patients which is again in contrast to the recent studies done [5,13,25]. Inflammatory markers are requested for prognostication of patients who might go into cytokine storm, reflecting a more severe type of disease. Majority of our patients had mild symptoms; the inflammatory response of their body might not have been triggered to increase their inflammatory markers.

In the interim guidelines on the screening, assessment and clinical management of pediatric patients with suspected or confirmed COVID-19 presented by the PPS and PIDSP last 2020, they mentioned that management of this disease in the pediatric age group is focused on providing best supportive care, management of co-existing conditions and treatment of possible bacterial co-infections [16]. In connection to this, majority of our subjects (85.6\%) were given supportive management and only $14.4 \%$ were given antibiotics. Those given with antibiotics were given supportive management also. No anti- viral medications or immunoglobulin were given as treatment to our patients. Majority of the children admitted with COVID- 19 recovered. Follow up was not done to those who were discharged at emergency department.

\section{Conclusion}

In conclusion, children with COVID-19 present with milder clinical course and rarely we encounter critical cases compared to infected adults. Early detection is the key to prevent further spread of this disease but a lot of our patients were asymptomatic hence diagnosis is a challenge for us pediatricians. Pediatric patients also present with different laboratory findings and variety of symptoms compared to what was already noted in the past studies. Most of our patients were sent home and was given supportive management. Almost all of the patients had distinct household contact to either a suspected or confirmed case of COVID-19. It is then important to emphasize proper quarantine precautions to the primary caregiver of these patients.

\section{Limitation and Recommendation}

These are the limitations for this study: First, several of the patients seen at the emergency department were not followed up after their initial consults. Therefore, we have no data regarding the duration of illness and occurrence of new symptoms for these patients. Long term outcome and sequela of these patients needs to be evaluated further. Second, the patients who followed up did not uniformly consulted at the same interval. This is important for us to also approximate the duration, and incubation period of this disease. Third, there were only a few severe or critical cases seen thus clinical course and management of these type of patients need to be further studied. This will also help in studying protective or risk factors of these children. Fourth, patients who were asymptomatic and with mild symptoms has fewer diagnostics done hence the results for the radiographic tests and laboratory tests were not representative of the whole sample population. 
Appendix

Appendix 1: Ethical clearance

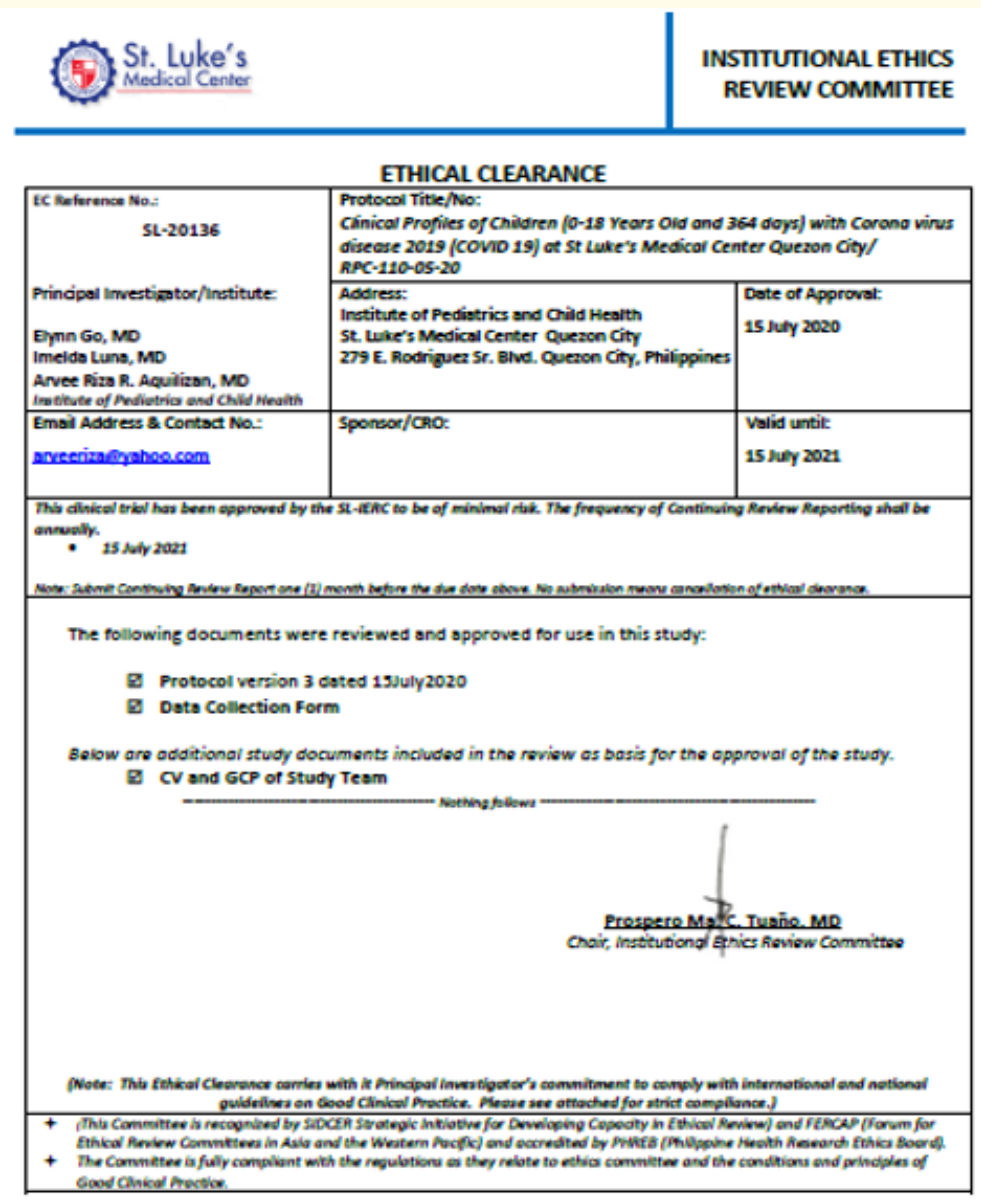

shateroes.19s

Figure 1

Appendix 2: Data collection form

Research title: Clinical Profiles of Children (0 - 18 Years Old) with Corona virus disease 2019 (COVID 19) at St Luke's Medical Center Quezon City.

\begin{tabular}{|l|c|c|}
\hline Investigators: & Name and Signature & Unit/Position \\
\hline Project Leader/s: & $\begin{array}{c}\text { Arvee Riza R. Aquilizan, } \\
\text { MD }\end{array}$ & Pediatric Resident \\
\hline $\begin{array}{l}\text { Co-Project } \\
\text { Leader/s: }\end{array}$ & Elynn Go, MD & Technical Adviser \\
\hline & Imelda Luna, MD & Content Adviser \\
\hline $\begin{array}{l}\text { Inst./Dept./Cen- } \\
\text { ter/Group: }\end{array}$ & $\begin{array}{c}\text { Institute of Pediatrics } \\
\text { and Child Health }\end{array}$ & \\
\hline
\end{tabular}

Patient Code

Date (MM/DD/YYYY):

\begin{tabular}{|l|l|l|}
\hline Screening: & Yes & No \\
\hline Inclusion Criteria & & \\
\hline $0-18$ years old & & \\
\hline Diagnosed with Corona Virus Disease 2019 & & \\
\hline Confirmed by COVID test PCR & & \\
\hline $\begin{array}{l}\text { If any inclusion criteria are ticked NO then the pa- } \\
\text { tient is not eligible for the study. }\end{array}$ & & \\
\hline
\end{tabular}




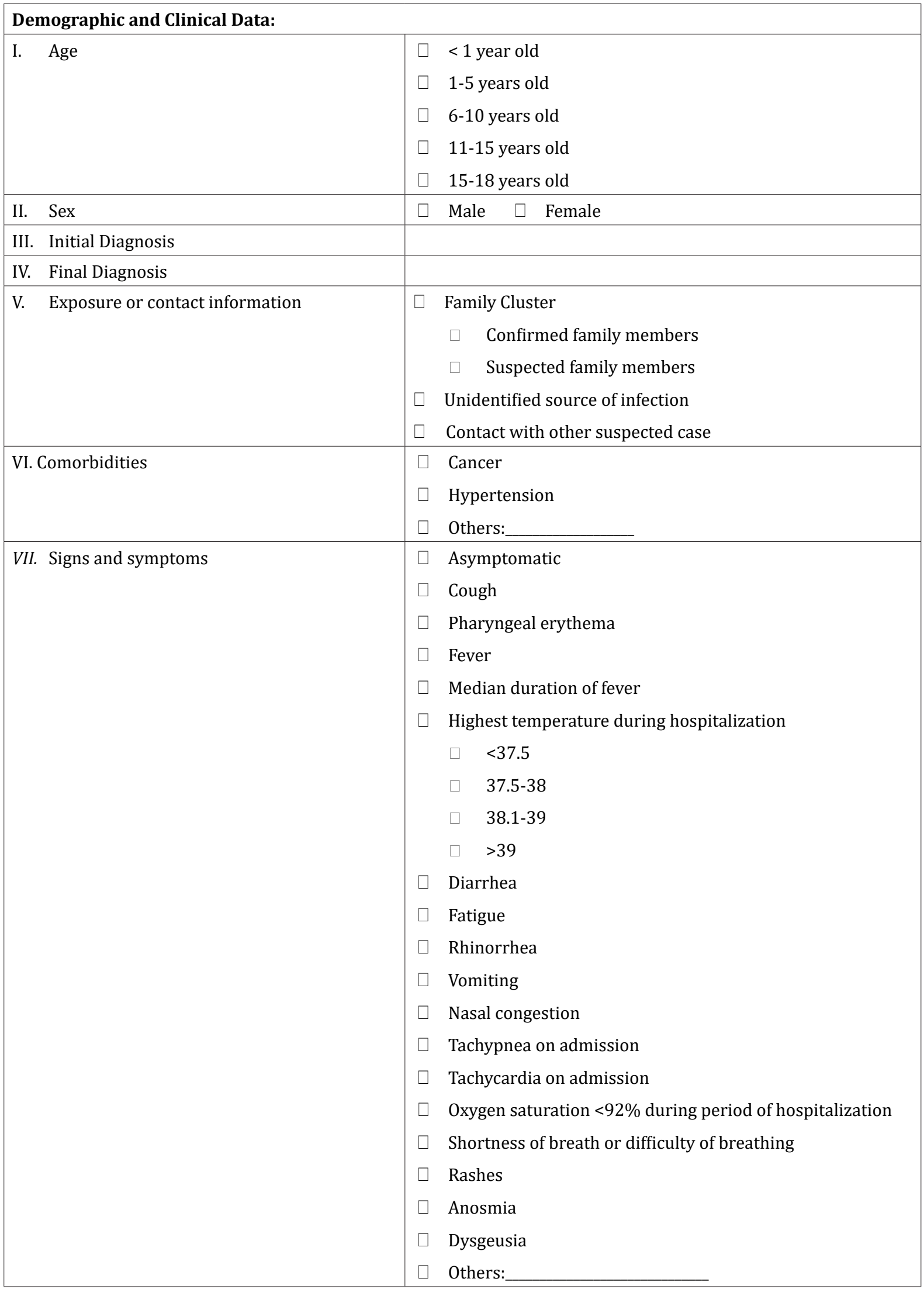




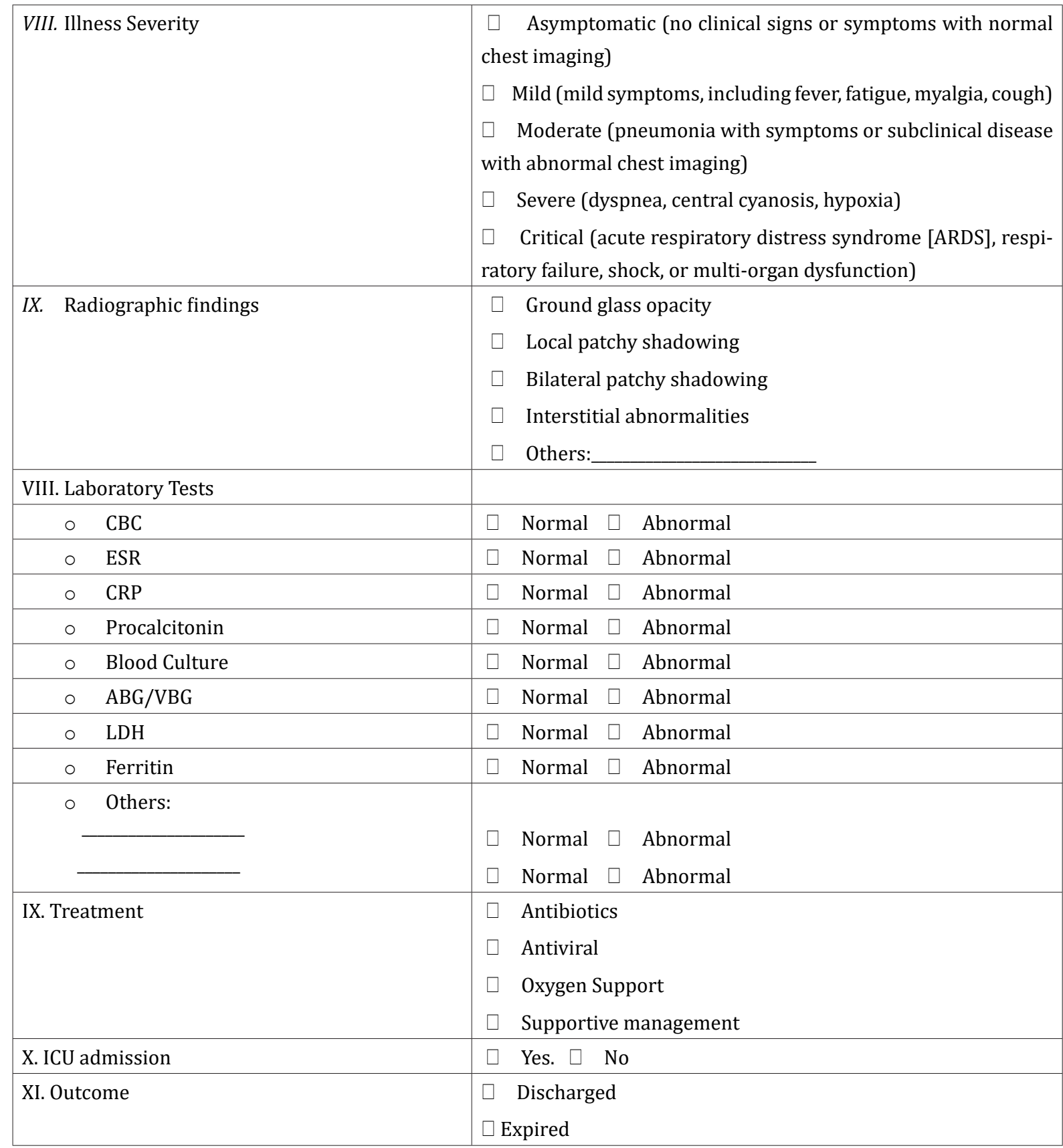

Completed by:

Name Signature Date

Appendix 3: First notice of action 


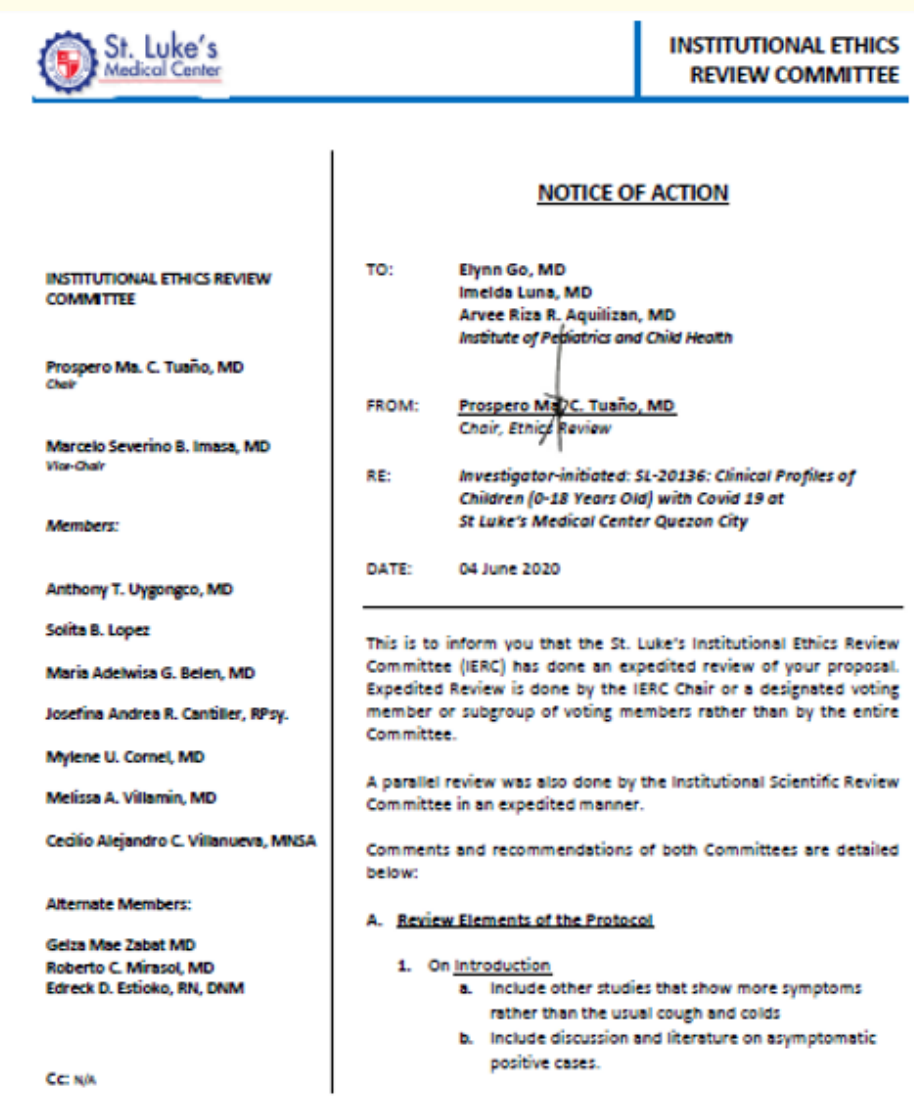

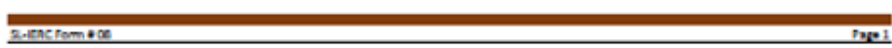

\begin{tabular}{l|r} 
(9). St. Luke's & $\begin{array}{r}\text { INSTITUTIONAL ETHICS } \\
\text { REVIEW COMMITITEE }\end{array}$ \\
\hline
\end{tabular}

\footnotetext{
2. On eliectives profie will be the focus of interest, such as riax factors, progroztic factors, or predictor varisbles.

b. Organize this section into General osjective ond Specihic Objectives, or Primary and Secondory objectives. 


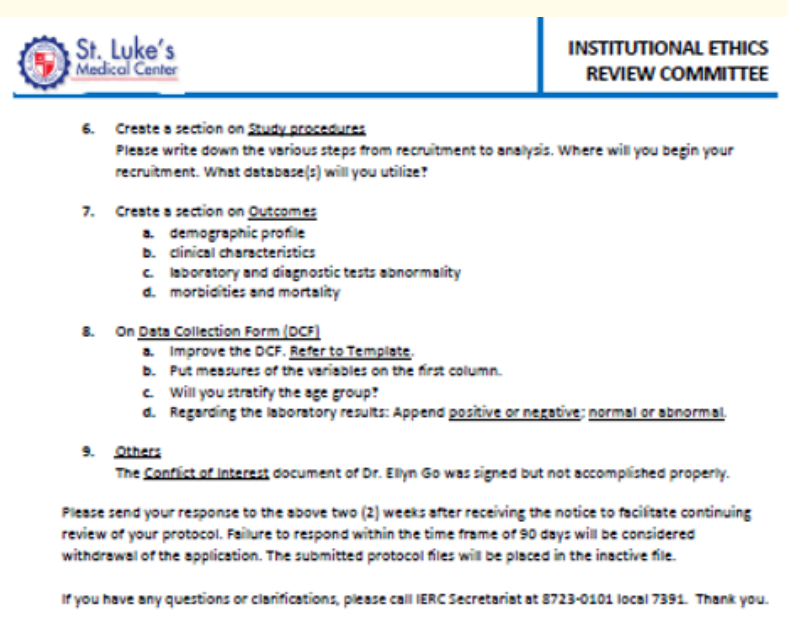

Figure 2

Appedix 4: Second notice of action

\begin{tabular}{l|l} 
9. St. Luke's & $\begin{array}{c}\text { INSTIUTIONAL ETHICS } \\
\text { REVIEW COMMITITEE Center }\end{array}$ \\
\hline
\end{tabular}

\begin{tabular}{|c|c|c|}
\hline \multirow{4}{*}{ 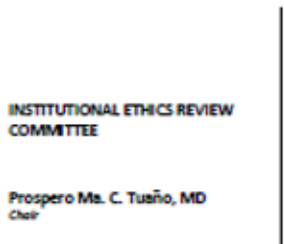 } & \multicolumn{2}{|r|}{ NOTICE OF ACTION } \\
\hline & то: & 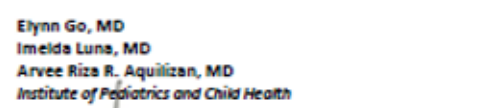 \\
\hline & & \\
\hline & FROM: & 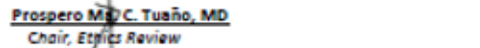 \\
\hline \multicolumn{3}{|l|}{ 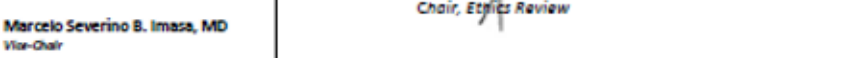 } \\
\hline \multirow[t]{2}{*}{ Member: } & RE: & 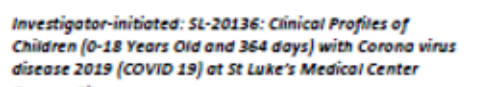 \\
\hline & & Quezon city \\
\hline Solits B. Loper & \multicolumn{2}{|c|}{ DATE: 14 Jur/ 2020} \\
\hline Maris Adewisa G. Belen, MD & & \\
\hline 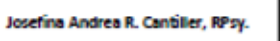 & \multirow{3}{*}{\multicolumn{2}{|c|}{ 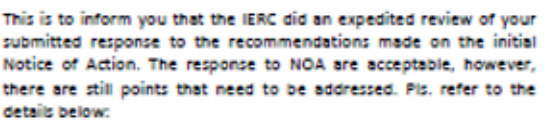 }} \\
\hline mprene U. Comel, MD & & \\
\hline Melizsa A. viltomin Mo & & \\
\hline 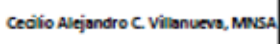 & \multirow{4}{*}{\multicolumn{2}{|c|}{ 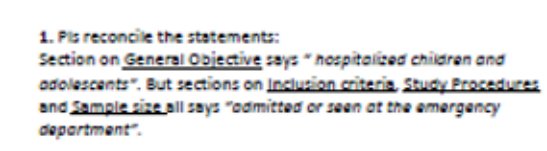 }} \\
\hline & & \\
\hline Antemate Members: & & \\
\hline \multirow{2}{*}{ 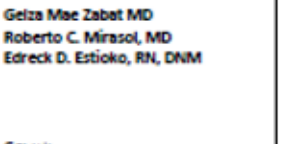 } & & \\
\hline & \multicolumn{2}{|r|}{ 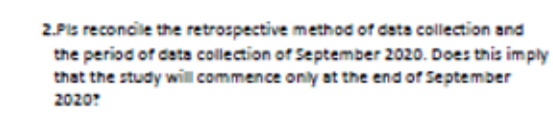 } \\
\hline \multirow[t]{2}{*}{ cen: } & \multicolumn{2}{|r|}{ 3.8eing a case series, renove the term "conort". } \\
\hline & \multicolumn{2}{|r|}{ 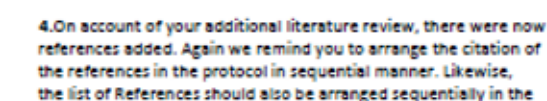 } \\
\hline
\end{tabular}



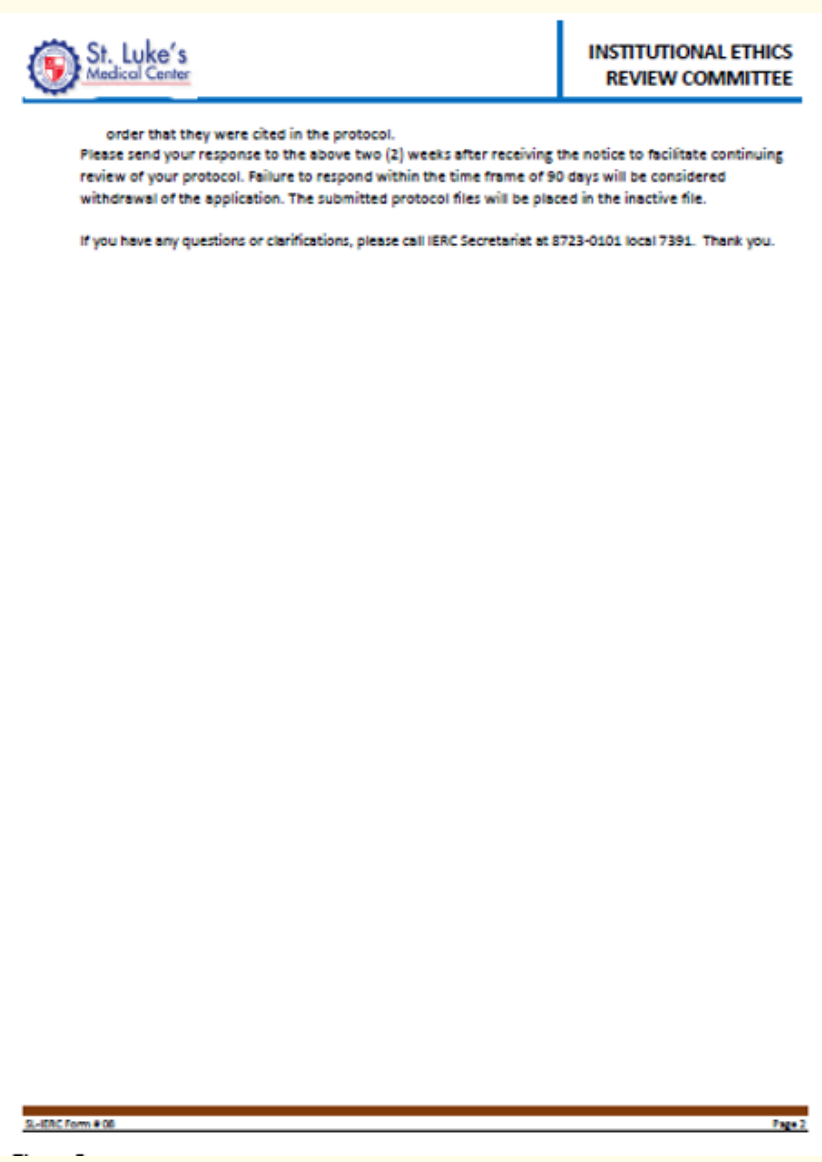

$-\cdot-$

Figure 3

\section{Bibliography}

1. Boger B., et al. "Systematic review with meta-analysis of the accuracy of diagnostic tests for COVID-19". American Journal of Infection Control (2020).

2. Borges L., et al. "COVID-19 and Neutrophils: The Relationship between Hyperinflammation and Neutrophil Extracellular Traps" (2020).

3. CDC COVID-19 Response Team. Coronavirus Disease 2019 in Children - United States (2020).

4. Chang TH., et al. "Clinical characteristics and diagnostic challenges of pediatric COVID-19: A systematic review and metaanalysis". Journal of the Formosan Medical Association (2020).
5. Chao J., et al. "Clinical Characteristics and Outcomes of Hospitalized and Critically Ill Children and Adolescents with Coronavirus Disease 2019 (COVID-19) at a Tertiary Care Medical Center in New York City". The Journal of Pediatrics (2020).

6. Corman VM., et al. "Detection of 2019 novel coronavirus (2019-nCoV) by real-time RT-PCR". Euro Surveill 25 (2020): 2000045.

7. Coronavirus Disease 2019; Information for Pediatric Healthcare Providers (2020).

8. COVID-19 CASE TRACKER (2020).

9. DOH Ro. Revised Administrative Order No. 2020-0012 “Guidelines for the Inclusion of the Coronavirus Disease 2019 (COVID-19) in the List of Notifiable Diseases for Mandatory Reporting to the Department of Health" (2020). 
10. Dong Y., et al. "Epidemiological Characteristics of 2143 Pediatric Patients With 2019 Coronavirus Disease in China”. Pediatrics (2019).

11. Guan W., et al. "Clinical characteristics of coronavirus disease 2019 in China". The New England Journal of Medicine (2019).

12. Han MS., et al. "Clinical Characteristics and Viral RNA Detection in Children With Coronavirus Disease 2019 in the Republic of Korea". JAMA Pediatrics (2020).

13. Hoang A., et al. "COVID- 19 in 7780 pediatric patients: A systematic review”. Elsevier (2020).

14. Holborow A., et al. "The clinical sensitivity of a single SARSCoV-2 upper respiratory tract RT-PCR test for diagnosing COVID-19 using convalescent antibody as a comparator". Royal College of Physician (2020).

15. Hughes $\mathrm{H}$ and Kahl L. "The Harriet Lane Handbook 21st edition. Philadelpia: ELSEVIER (2018).

16. Interim Guidelines on the Screening, Assessment and Clinical Management of Pediatric Patients with Suspected or Confirmed Coronavirus Disease 2019 (COVID-19). Philippine Pediatric Society and Pediatric Infectious Disease Society of the Philippines (2020).

17. Ji LN., et al. "Clinical features of pediatric patients with COVID-19: a report of two family cluster cases". World Journal of Pediatrics: WJP (2020).

18. Lechien J., et al. "Olfactory and gustatory dysfunctions as a clinical presentation of mild-to-moderate forms of the coronavirus disease (COVID-19): a multicenter European study". European Archives of Oto-Rhino-Laryngology (2020).

19. Livingston E and Bucher K. "Coronavirus Disease 2019 (COVID-19) in Italy". JAMA: The Journal of the American Medical Association (2020).

20. Lu X., et al. "Chinese Pediatric Novel Coronavirus Study Team. SARS-CoV-2 infection in children". The New England Journal of Medicine 382.17 (2020): 1663-1665.

21. World Health Organization Coronavirus disease (COVID-19) Dashboard (2020).

22. Tagarro A., et al. "Screening and Severity of Coronavirus Disease 2019 (COVID-19) in Children in Madrid, Spain”. JAMA Pediatrics (2020).
23. World Health Organization. Retrieved from Coronavirus disease (COVID-19) in the Philippines (2020).

24. Wu Z and McGoogan JM. "Characteristics of and Important Lessons From the Coronavirus Disease 2019 (COVID-19) Outbreak in China: Summary of a Report of 72314 Cases From the Chinese Center for Disease Control and Prevention". JAMA: The Journal of the American Medical Association (2020).

25. Xia W., et al. "Clinical and CT features in pediatric patients with COVID-19 infection: Different points from adults". Pediatric Pulmonology (2020).

\section{Volume 4 Issue 10 October 2021}

(C) All rights are reserved by Arvee Riza R Aquilizan., et al. 